\title{
BOUNDED SOLUTIONS FOR FUZZY INTEGRAL EQUATIONS
}

\author{
D. N. GEORGIOU and I. E. KOUGIAS
}

Received 1 August 2001

We study conditions under which the solutions of a fuzzy integral equation are bounded.

2000 Mathematics Subject Classification: 54A40, 26E50.

1. Introduction. The concept of set-valued functions and their calculus [2] were found useful in some problems in economics [3], as well as in control theory [17]. Later on, the notion of $H$-differentiability was introduced by Puri and Ralesku in order to extend the differential of set-valued functions to that of fuzzy functions [29]. This in turn led Seikkala [30] to introduce the notion of fuzzy derivative, which is a generalization of the Hukuhara derivative and the fuzzy integral, which is the same as that proposed by Dubois and Prade [7, 8]. A natural consequence of the above was the study of fuzzy differential and integral equations, see $[5,9,10,11,12,18,19,20$, $21,23,24,25,26,28,29,30,31,32,33]$.

Fixed point theorems for fuzzy mappings, an important tool for showing existence and uniqueness of solutions to fuzzy differential and integral equations, have recently been proved by various authors, see [1, 4, 13, 14, 15, 16, 22, 27]. In particular, in [22] Lakshmikantham and Vatsala proved the existence of fixed points to fuzzy mappings, using theory of fuzzy differential equations. Finally, stability criteria for the solutions of fuzzy differential systems are given in [21].

In this paper, we examine conditions under which all the solutions of the fuzzy integral equation

$$
x(t)=\int_{0}^{t} G(t, s) x(s) d s+f(t)
$$

and the special case

$$
x(t)=\int_{0}^{t} k(t-s) x(s) d s+f(t)
$$

are bounded.

These fuzzy integral equations are proved useful when studying observability of fuzzy dynamical control systems, see [6].

2. Preliminaries. By $P_{k}\left(\mathbb{R}^{n}\right)$, we denote the family of all nonempty compact convex subsets of $\mathbb{R}^{n}$. 
For $A, B \in P_{k}\left(\mathbb{R}^{n}\right)$, the Hausdorff metric is defined by

$$
d(A, B)=\max \left\{\sup _{a \in A} \inf _{b \in B}\|a-b\|, \sup _{b \in B} \inf _{a \in A}\|a-b\|\right\}
$$

A fuzzy set in $\mathbb{R}^{n}$ is a function with domain $\mathbb{R}^{n}$ and values in $[0,1]$, that is an element of $[0,1]^{\mathbb{R}^{n}}$ (see $\left.[35,34]\right)$.

Let $u \in[0,1]^{\mathbb{R}^{n}}$, the a-level set is

$$
\begin{aligned}
{[u]^{a} } & =\left\{x \in \mathbb{R}^{n}: u(x) \geq a\right\}, \quad a \in(0,1], \\
{[u]^{0} } & =\mathrm{Cl}\left(\left\{x \in \mathbb{R}^{n}: u(x)>0\right\}\right) .
\end{aligned}
$$

By $E^{n}$, we denote the family of all fuzzy sets $u \in[0,1]^{\mathbb{R}^{n}}$ (see $[18,29,35]$ ), for which:

(i) $u$ is normal, that is, there exists an element $x_{0} \in \mathbb{R}^{n}$, such that $u\left(x_{0}\right)=1$,

(ii) $u$ is fuzzy convex,

(iii) $u$ is uppersemicontinuous,

(iv) $[u]^{0}$ is compact.

Let $u \in E^{n}$. Then for each $a \in(0,1]$ the $a$-level set $[u]^{a}$ of $u$ is a nonempty compact convex subset of $\mathbb{R}^{n}$, that is, $u \in P_{k}\left(\mathbb{R}^{n}\right)$. Also $[u]^{0} \in P_{k}\left(\mathbb{R}^{n}\right)$.

Let

$$
D: E^{n} \times E^{n} \longrightarrow[0, \infty), \quad D(u, v)=\sup \left\{d\left([u]^{a},[v]^{a}\right): a \in[0,1]\right\}
$$

where $d$ is the Hausdorff metric for nonempty compact convex subsets of $\mathbb{R}^{n}$ (see [18]).

\section{Main results}

Notation 3.1. By $\hat{0} \in E^{n}$, we denote the fuzzy set for which $\hat{0}(x)=1$ if $x=0$ and $\hat{0}(x)=0$ if $x \neq 0$.

Definition 3.2. A mapping $x: T \rightarrow E^{n}$ is bounded, where $T$ is an interval of the real line, if there exists an element $r>0$, such that

$$
D(x(t), \hat{0})<r, \quad \forall t \in T \text {. }
$$

THEOREM 3.3. Suppose that $f:[0,+\infty) \rightarrow E^{n}$ with $D(f(t), \hat{0}) \leq M$, and $G: \Delta \rightarrow \mathbb{R}$ is continuous, where $\Delta=\{(t, s): 0 \leq s \leq t<\infty\}$. If there exists $m<1$ with $\int_{0}^{t}|G(t, s)| d s$ $\leq m$, for $t \in[0,+\infty)$, then all solutions of the fuzzy integral equation

$$
x(t)=\int_{0}^{t} G(t, s) x(s) d s+f(t),
$$

are bounded. 
Proof. Let $x(t)$ be an unbounded solution of (3.2). Then for every $r>0$, there exists an element $t_{1} \in(0, \infty)$, such that

$$
D(x(s), \hat{0})<r, \quad \forall s \in\left[0, t_{1}\right), \quad D\left(x\left(t_{1}\right), \hat{0}\right)=r .
$$

Clearly, we can find a positive number $r$ with

$$
M+m r<r
$$

By (3.3), (3.4), and the assumptions of the theorem we have

$$
\begin{aligned}
r & =D\left(x\left(t_{1}\right), \hat{0}\right) \\
& =D\left(\int_{0}^{t_{1}} G\left(t_{1}, s\right) x(s) d s+f\left(t_{1}\right), \hat{0}\right) \\
& \leq D\left(\int_{0}^{t_{1}} G\left(t_{1}, s\right) x(s) d s, \hat{0}\right)+D\left(f\left(t_{1}\right), \hat{0}\right) \\
& \leq \int_{0}^{t_{1}} D\left(G\left(t_{1}, s\right) x(s), \hat{0}\right) d s+M \quad(\text { see }[19, \text { Theorem 4.3]) } \\
& \leq \int_{0}^{t_{1}}\left|G\left(t_{1}, s\right)\right| D(x(s), \hat{0}) d s+M \quad \text { (by the definition of } D, \text { see [19]) } \\
& \leq M+m r<r,
\end{aligned}
$$

which is a contradiction. Thus $x(t)$ is bounded.

THEOREM 3.4. Suppose that $f:[0, \infty) \rightarrow E^{n}$ and $k:[0, \infty) \rightarrow \mathbb{R}$ are continuous and that there exist constants $A, B$, and $a>0$ with $0<B<1$ and

$$
D(f(t), \hat{0}) \leq A e^{-a t}, \quad \int_{0}^{t}|k(t-s)| d s \leq B e^{-a t} .
$$

Then, every solution of the fuzzy integral equation

$$
x(t)=\int_{0}^{t} k(t-s) x(s) d s+f(t)
$$

is bounded.

Proof. Let $x(t)$ be an unbounded solution of (3.7). Then for every $r>0$, there exists an element $t_{1} \in(0, \infty)$, such that

$$
D(x(s), \hat{0})<r, \quad \forall s \in\left[0, t_{1}\right), \quad D\left(x\left(t_{1}\right), \hat{0}\right)=r .
$$


Clearly, we can find a positive number $r$ with

$$
A+B r<r
$$

By (3.6), (3.7), and (3.8) we have

$$
\begin{aligned}
r & =D\left(x\left(t_{1}\right), \hat{0}\right) \\
& =D\left(\int_{0}^{t_{1}} k\left(t_{1}-s\right) x(s) d s+f\left(t_{1}\right), \hat{0}\right) \\
& \leq D\left(\int_{0}^{t_{1}} k\left(t_{1}-s\right) x(s) d s, \hat{0}\right)+D\left(f\left(t_{1}\right), \hat{0}\right) \\
& \leq \int_{0}^{t_{1}} D\left(k\left(t_{1}-s\right) x(s), \hat{0}\right) d s+D\left(f\left(t_{1}\right), \hat{0}\right) \quad \text { (see [19, Theorem 4.3]) } \\
& \leq \int_{0}^{t_{1}}\left|k\left(t_{1}-s\right)\right| D(x(s), \hat{0}) d s+D\left(f\left(t_{1}\right), \hat{0}\right) \quad \text { (by the definition of } D, \text { see [19]) } \\
& \leq A e^{-a t_{1}}+B e^{-a t_{1}} r \\
& \leq A e^{-a t_{1}}+B e^{-a t_{1}} r
\end{aligned}
$$

and thus

$$
e^{a t_{1}} r<A+B r<r,
$$

which is a contradiction. Thus, $x(t)$ is bounded.

REMARK 3.5. Now, since the initial value problem

$$
x^{\prime}(t)=f(t, x(t)), \quad t \in T, x(0)=x_{0},
$$

where $f: T \times E^{n} \rightarrow E^{n}$ is continuous, it is equivalent to the integral equation

$$
x(t)=x_{0}+\int_{0}^{t} f(s, x(s)) d s, \quad t \in[0, b] \quad \text { (see [20, Lemma 3.1]). }
$$

If for the map $f: T \times E^{n} \rightarrow E^{n}$ the conditions of Theorem 3.3 or 3.4 hold true, then all the solutions of the initial value problem (3.12) are bounded.

4. Conclusion. In this paper, using a Gronwall type inequality, we give conditions under which the fuzzy integral equations (3.2) and (3.7) possess only bounded solutions. Consequently, this implies that the Cauchy problem (3.12) possesses only bounded solutions as well. It appears that, these fuzzy equations are useful when one studies the observability of fuzzy dynamical control systems. We also think that, our results can be of use in studying stability of fuzzy differential equations and fuzzy differential systems. 


\section{REFERENCES}

[1] S. C. Arora and V. Sharma, Fixed point theorems for fuzzy mappings, Fuzzy Sets and Systems 110 (2000), no. 1, 127-130.

[2] R. J. Aumann, Integrals of set-valued functions, J. Math. Anal. Appl. 12 (1965), 1-12.

[3] R. J. Aumann and M. Perles, A variational problem arising in economics, J. Math. Anal. Appl. 11 (1965), 488-503.

[4] R. K. Bose and D. Sahani, Fuzzy mappings and fixed point theorems, Fuzzy Sets and Systems 21 (1987), no. 1, 53-58.

[5] J. J. Buckley and T. Feuring, Fuzzy differential equations, Fuzzy Sets and Systems 110 (2000), no. 1, 43-54.

[6] Z. Ding, M. Ma, and A. Kandel, On the observability of fuzzy dynamical control systems. I, Fuzzy Sets and Systems 111 (2000), no. 2, 225-236.

[7] D. Dubois and H. Prade, Towards fuzzy differential calculus. I. Integration of fuzzy mappings, Fuzzy Sets and Systems 8 (1982), no. 1, 1-17.

[8] _ Towards fuzzy differential calculus. II. Integration on fuzzy intervals, Fuzzy Sets and Systems 8 (1982), no. 2, 105-116.

[9] M. Friedman, M. Ma, and A. Kandel, Numerical solutions of fuzzy differential and integral equations, Fuzzy Sets and Systems 106 (1999), no. 1, 35-48.

[10] M. Friedman, M. Ming, and A. Kandel, Numerical methods for calculating the fuzzy integral, Fuzzy Sets and Systems 83 (1996), no. 1, 57-62.

[11] Solutions to fuzzy integral equations with arbitrary kernels, Internat. J. Approx. Reason. 20 (1999), no. 3, 249-262.

[12] D. N. Georgiou and I. E. Kougias, On fuzzy Fredholm and Volterra integral equations, J. Fuzzy Math. 9 (2001), no. 4, 943-951.

[13] V. Gregori and S. Romaguera, A generalization of common fixed point theorems for pairs of fuzzy mappings in quasi-metric spaces, in preparation.

[14]__ Common fixed point theorems for pairs of fuzzy mappings, Indian J. Math. 41 (1999), no. 1, 43-54.

[15] _ Fixed point theorems for fuzzy mappings in quasi-metric spaces, Fuzzy Sets and Systems 115 (2000), no. 3, 477-483.

[16] S. Heilpern, Fuzzy mappings and fixed point theorem, J. Math. Anal. Appl. 83 (1981), no. 2, 566-569.

[17] H. Hermes, Calculus of set valued functions and control, J. Math. Mech. 18 (1969), 47-59.

[18] O. Kaleva, On the convergence of fuzzy sets, Fuzzy Sets and Systems 17 (1985), no. 1, 53-65.

[19] _ Fuzzy differential equations, Fuzzy Sets and Systems 24 (1987), no. 3, 301-317.

[20] _ The Cauchy problem for fuzzy differential equations, Fuzzy Sets and Systems 35 (1990), no. 3, 389-396.

[21] V. Lakshmikantham, Uncertain systems and fuzzy differential equations, J. Math. Anal. Appl. 251 (2000), no. 2, 805-817.

[22] V. Lakshmikantham and A. S. Vatsala, Existence of fixed points of fuzzy mappings via theory of fuzzy differential equations, J. Comput. Appl. Math. 113 (2000), no. 1-2, 195-200.

[23] M. Ma, M. Friedman, and A. Kandel, Numerical solutions of fuzzy differential equations, Fuzzy Sets and Systems 105 (1999), no. 1, 133-138.

[24] J. J. Nieto, The Cauchy problem for continuous fuzzy differential equations, Fuzzy Sets and Systems 102 (1999), no. 2, 259-262.

[25] J. Y. Park and H. K. Han, Fuzzy differential equations, Fuzzy Sets and Systems 110 (2000), no. 1, 69-77.

[26] J. Y. Park, H. K. Han, and K. H. Son, Fuzzy differential equation with nonlocal condition, Fuzzy Sets and Systems 115 (2000), no. 3, 365-369.

[27] J. Y. Park and J. U. Jeong, Fixed point theorems for fuzzy mappings, Fuzzy Sets and Systems 87 (1997), no. 1, 111-116. 
[28] _ A note on fuzzy integral equations, Fuzzy Sets and Systems 108 (1999), no. 2, 193-200.

[29] M. L. Puri and D. A. Ralescu, Differentials of fuzzy functions, J. Math. Anal. Appl. 91 (1983), no. 2, 552-558.

[30] S. Seikkala, On the fuzzy initial value problem, Fuzzy Sets and Systems 24 (1987), no. 3, 319-330.

[31] S. Song and C. Wu, Existence and uniqueness of solutions to Cauchy problem of fuzzy differential equations, Fuzzy Sets and Systems 110 (2000), no. 1, 55-67.

[32] P. V. Subrahmanyam and S. K. Sudarsanam, A note on fuzzy Volterra integral equations, Fuzzy Sets and Systems 81 (1996), no. 2, 237-240.

[33] C. Wu, S. Song, and E. S. Lee, Approximate solutions, existence, and uniqueness of the Cauchy problem of fuzzy differential equations, J. Math. Anal. Appl. 202 (1996), no. 2, 629-644.

[34] L. A. Zadeh, Fuzzy sets, Information and Control 8 (1965), 338-353.

[35] H. J. Zimmermann, Fuzzy Theory and Its Applications, 2nd ed., Kluwer Academic Publishers, Massachusetts, 1991.

D. N. Georgiou: UniVersity of PATRAs, FACUlty of SCIENCES, DePARTMENT OF MATHEMATICS, 26500 PATRAS, GREECE

E-mail address: georgiou@math.upatras.gr

I. E. Kougias: Technological Educational Institute of Epirus, School of Business Administration And Economics, Department of Accounting, 48100 Preveza, Greece E-mail address: kougias@teiep.gr 


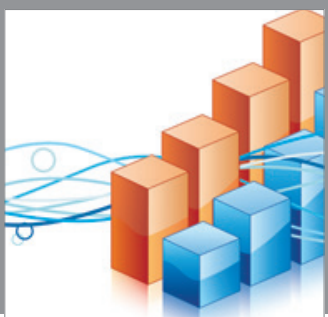

Advances in

Operations Research

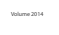

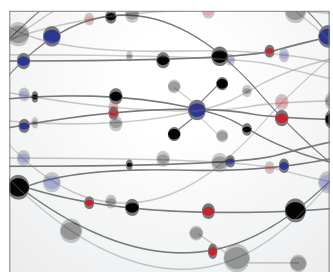

\section{The Scientific} World Journal
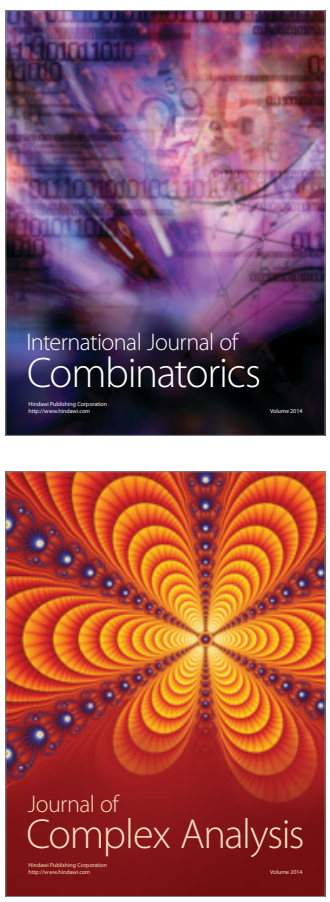

International Journal of

Mathematics and

Mathematical

Sciences
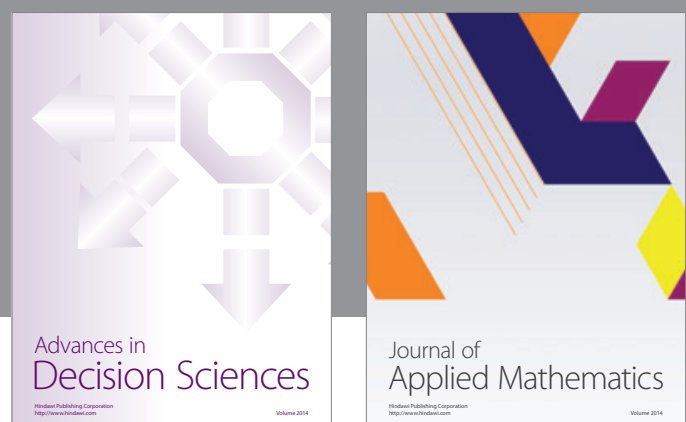

Journal of

Applied Mathematics
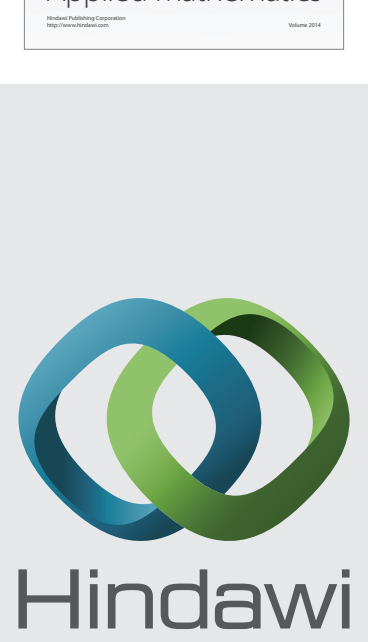

Submit your manuscripts at http://www.hindawi.com
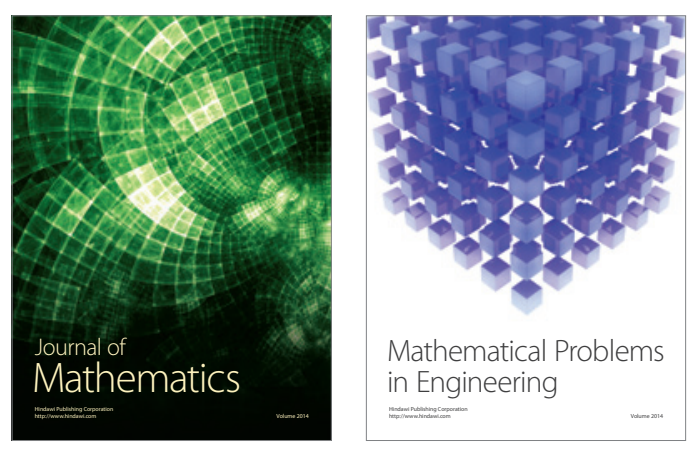

Mathematical Problems in Engineering
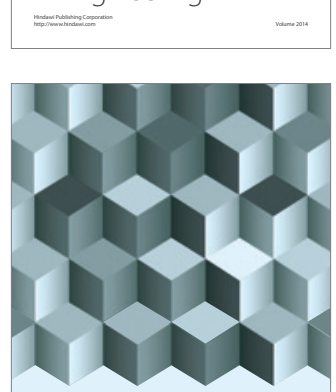

Journal of

Function Spaces
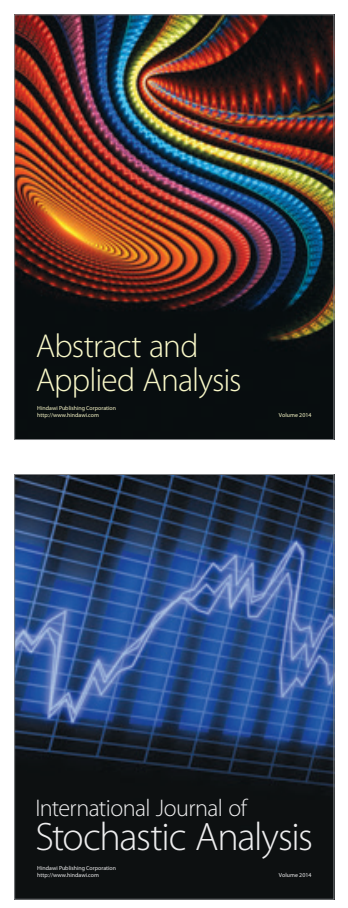

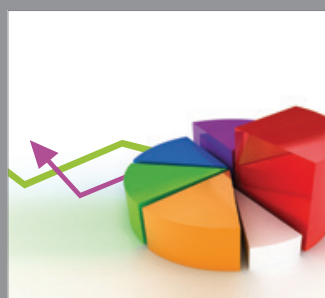

ournal of

Probability and Statistics

Promensencen
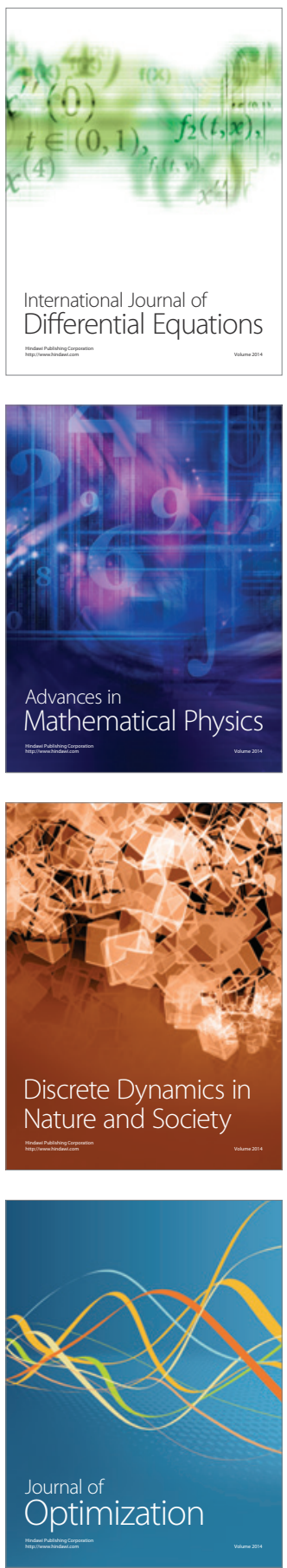